\title{
Editorial
}

\section{Stem Cell - A Miraculous Cell Creating a Ray of Future Hope}

\section{Introduction:}

Ultimately, every cell in the human body can be traced back to a fertilized egg that came into existence from the union of ovum and sperm. But the body is made up of over 200 different types of cells, not just one. All of these cell types come from a pool of cells in the early embryo, called stem cells. $^{1}$

Importance of the stem cell:

Stem cells have the remarkable potentiality to develop into many different cell types in the body during early life and growth. In addition, in many tissues they serve as a sort of internal repair system, dividing essentially without limit to replenish other cells as long as the person or animal is still alive. When a stem cell divides, each new cell has the potentially either to remain a stem cell or become another type of cell with a more specialized function, such as a muscle cell, a red blood cell, or a brain cell. ${ }^{2}$

\section{Unique property of the stem cell:}

Stem cells are distinguished from other cell types by two important characteristics. Firstly, they are unspecialized cells capable of renewing themselves through cell division, sometimes after long periods of inactivity. Secondly, under certain physiologic or experimental conditions, they can be induced to become tissue- or organ-specific cells with special functions. In some organs, such as the gut and bone marrow, stem cells regularly divide to repair and replace worn out or damaged tissues. In other organs, however, such as the pancreas and the heart, stem cells only divide under special conditions. $^{2}$

\section{Types of the stem cell:}

1. Embryonic stem cells.

2. Adult stem cells.

3. Umbilical cord blood stem cells.

\section{Embryonic stem cells:}

As their name suggests, they are derived from embryos. Most embryonic stem cells are derived from embryos that develop from eggs that have been fertilized in vitro-in an in vitro fertilization clinicand then donated for research purposes with informed consent of the donors. ${ }^{3}$ They are not derived from eggs fertilized in a woman's body. They have the potential to produce all of the body's cell types. ${ }^{1} \mathrm{~A}$ blastocyst is a pre-implantation embryo that develops 5 days after the fertilization of an egg by a sperm. It contains all the material necessary for the development of complete human being. In the interior of the blastocyst the inner cell mass, this is composed of $30-34$ cells that are referred to by scientists as pluripotent because they can differentiate into all of the cell types of the body. ${ }^{1}$

\section{Adult stem cells:}

An adult stem cell is thought to be an undifferentiated cell, found among differentiated cells in a tissue or organ that can renew itself and can differentiate to yield some or all of the major specialized cell types of the tissue or organ. The primary roles of adult stem cells in a living organism are to maintain and repair the tissue in which they are found. Scientists also use the term somatic stem cell instead of adult stem cell, where somatic refers to cells of the body (not the germ cells, sperm or eggs). Unlike embryonic stem cells, which are defined by their origin (the inner cell mass of the blastocyst), the origin of adult stem cells in some mature tissues is still under investigation. ${ }^{4}$

Adult stem cells are hidden deep within organs, surrounded by millions of ordinary cells, and may help replenish some of the body's cells when needed. In fact, some adult stem cells are currently being used in therapies. They have been found in several organs that need a constant supply of cells, 
such as the blood, skin, and lining of the G.I.T., and have also been found in surprising places like the brain, which is not known to readily replenish its cells. ${ }^{1}$ Unlike embryonic stem cells, adult stem cells are already somewhat specialized. For example, blood stem cells normally only give rise to many types of blood cells and nerve stem cells can only make the various types of brain cells. However, recent study with adult stem cells suggests that some adult stem cells might be more flexible than previously thought, and may be made to produce a wider variety of cell types, but the results not proven and have not been demonstrated yet with human cells. ${ }^{1}$

\section{Umbilical cord blood stem cells:}

Umbilical cord blood is the blood left over in the placenta and in the umbilical cord after the birth of the baby, after the cord has been detached from the newborn. The cord blood contains some specialized cell called stem cells, including hematopoietic cells. Umbilical cord blood is well-recognized to be useful for treating hematopoietic and genetic disorders. ${ }^{5}$ Removing the umbilical cord blood is not harmful to the baby and the blood would normally be thrown away as medical waste. The use of cord blood stem cells in treating conditions such as brain injury and Type 1 Diabetes $\mathbf{6}$ is already being studied in humans, and earlier stage research is being conducted for treatments of stroke ${ }^{7,8}$, and hearing loss. ${ }^{9}$ However, apart from blood disorders, the use of cord blood for other diseases is not a routine clinical modality and remains a major challenge for the stem cell community. Researchers are still exploring the use of cord blood stem cells in the following regenerative medicine applications: Brain Injury, Cerebral Palsy Type 1 Diabetes, Heart Disease etc. ${ }^{10}$

\section{Differentiation between Embryonic and Adult stem cells:}

Human embryonic and adult stem cells each have advantages and disadvantages regarding potential use for cell-based regenerative therapies. One major difference between adult and embryonic stem cells is their different abilities in the number and type of differentiated cell types they can become. Embryonic stem cells can become all cell types of the body because they are pluripotent. Adult stem cells are thought to be limited to differentiating into different cell types of their tissue of origin. ${ }^{11}$ Adult stem cells are not found in all tissues \& difficult to identify, isolate, maintain, and grow in the laboratory ${ }^{1}$. However, ethical implication arises in case of Embryonic stem cell therapy; because for Embryonic stem cells it requires destruction of human blastocysts. ${ }^{1}$ Donation of blastocysts requires informed consent from the donor. ${ }^{1,3}$ Whereas no major ethical concerns have been raised in case of Adult stem cell therapy. ${ }^{1}$

\section{Stem cell therapy at the present time:}

Several stem cell therapies are routinely used to treat disease today.

\section{These include:}

1. Adult Stem Cell Transplant: Bone Marrow Stem Cells

2. Adult Stem Cell Transplant: Peripheral Blood Stem Cells

\section{Umbilical Cord Blood Stem Cell Transplant}

Adult Stem Cell Transplant: Bone Marrow Stem Cells

Possibly the best-known stem cell therapy to date is the bone marrow transplant, which is used to treat leukemia and other types of cancer, as well as various blood disorders. ${ }^{12}$

Leukemia is a cancer of white blood cells, or leukocytes. Like other blood cells, leukocytes are made in the bone marrow through a process that begins with multipotent adult stem cells. Mature leukocytes are released into the bloodstream, where they work to fight off infections in our bodies.

Leukemia results when leukocytes begin to grow and function abnormally, becoming cancerous. These abnormal cells cannot fight off infection, and they interfere with the functions of other organs.

Successful treatment for leukemia depends on getting rid of all the abnormal leukocytes in the patient, allowing healthy ones to grow in their place. One way to do this is through chemotherapy, which uses potent drugs to target and kill the abnormal cells. When chemotherapy alone can't eliminate 
them all, physicians sometimes turn to bone marrow transplants. ${ }^{12}$

In a bone marrow transplant, the patient's bone marrow stem cells are replaced with those from a healthy, matching donor. To do this, all of the patient's existing bone marrow and abnormal leukocytes are first killed using a combination of chemotherapy and radiation. Next, a sample of donor bone marrow containing healthy stem cells is introduced into the patient's bloodstream. ${ }^{12}$

If the transplant is successful, the stem cells will migrate into the patient's bone marrow and begin producing new, healthy leukocytes to replace the abnormal cells. ${ }^{11}$

Adult Stem Cell Transplant: Peripheral Blood Stem Cell (PBSC) Transplant

While most blood stem cells reside in the bone marrow, a small number are present in the bloodstream. These multipotent peripheral blood stem cells, or PBSCs, can be used just like bone marrow stem cells to treat leukemia, other cancers and various blood disorders. Since they can be obtained from drawn blood, PBSCs are easier to collect than bone marrow stem cells, which must be extracted from within bones. This makes PBSCs a less invasive treatment option than bone marrow stem cells. PBSCs are sparse in the bloodstream, however, so collecting enough to perform a transplant can pose a challenge. ${ }^{12}$

\section{Umbilical Cord Blood Stem Cell Transplant:}

Newborn infants no longer need their umbilical cords, so they have traditionally been discarded as a byproduct of the birth process. In recent years, however, the multipotent-stem-cell-rich blood found in the umbilical cord has proven useful in treating the same types of health problems as those treated using bone marrow stem cells and PBSCs. ${ }^{12}$

Umbilical cord blood stem cell transplants are less prone to rejection than either bone marrow or peripheral blood stem cells. ${ }^{12}$ This is probably because the cells have not yet developed the features that can be recognized and attacked by the recipient's immune system. Also, because umbilical cord blood lacks well-developed immune cells, there is less chance that the transplanted cells will attack the recipient's body, a problem called graft versus host disease. ${ }^{12}$

Both the versatility and availability of umbilical cord blood stem cells makes them a potent resource for transplant therapies. The first successful cord blood stem cell transplant was performed in 1988 in Paris, France. ${ }^{13}$ The patient was a boy with Fanconi anemia, a genetic and potentially life-threatening type of anemia. Cord blood stem cell transplants have now been given successfully to patients (mostly children) with some 70 diseases, including acute lymphocytic leukemia (also called "acute lymphoblastic leukemia," or ALL), acute myelogenous leukemia (AML), myelodysplastic syndromes (MDS), chronic myelogenous leukemia $(\mathrm{CML})$, juvenile chronic myelogenous leukemia (JCML), chronic lymphocytic leukemia (CLL), Hodgkin and non-Hodgkin lymphoma, neuroblastoma, thalassemia, severe combined immune deficiency (SCID), Wiskott-Aldrich syndrome, metabolic diseases such as diabetes mellitus, adrenoleukodystrophy and Hurler syndrome, and severe aplastic anemia. To date, more than 5,500 cord blood stem cell transplants from unrelated donors and several hundred from sibling donors have been performed worldwide.13 Cord blood stem cells give new hope to repair brain damage now a day. ${ }^{14}$

\section{Conclusion:}

In this new age of medical science, stem cell therapy can provide both help and hope to those patients suffering from diseases and conditions, which were not possible to treat before but treatable and to some extent even curable now. But, as it is a new advancement of medical science, people, who are not too familiar with it, try to use it for their own gain and benefit. Stem cell therapy will definitely open a new horizon in the medical science and undoubtedly creating a hope for the future generation.

\section{Sanjoy Kumar Chakraborty ${ }^{1}$, Laila Anjuman Banu $^{2}$}

1. Assistant Prof. Department of Anatomy, BGC Trust Medical College, Chittagong.

2. Professor Department of Anatomy, BSMMU, Dhaka. 


\section{References:}

1. www.national-academies.org. [accessed on 15. 2. 2011].

2. http://stemcells.nih.gov/info/basics/basics1. [accessed on 22. 2. 2011].

3. http://stemcells.nih.gov/info/basics/basics3. [accessed on 1.3. 2011].

4. http://stemcells.nih.gov/info/basics/basics4. [accessed on 12. 4. 2011].

5. Cairo MS, Wagner JE. "Placental and/or umbilical cord blood: an alternative source of hematopoietic stem cells for transplantation.". Blood 90 1997; 12: 4665-78

6. Haller MJ, etal.; Viener, HL; Wasserfall, C; Brusko, T; Atkinson, MA; Schatz, DA. "Autologous umbilical cord blood infusion for type 1 diabetes.". Exp. Hematol. 36 2008; 6: 710-15.

7. Vendrame M, et al. "Cord blood rescues strokeinduced changes in splenocyte phenotype and function.". Exp. Neurol. 2006;199: 191-200.
8. Vendrame $\mathrm{M}$, et al. "Anti-inflammatory effects of human cord blood cells in a rat model of stroke.". Stem Cells Dev. 2005; 14: 595-604.

9. Revoltella RP, et al. "Cochlear repair by transplantation of human cord blood CD133+ cells to nod-scid mice made deaf with kanamycin and noise.". Cell Transplant. 2008;17: 665-78.

10. http://en.wikipedia.org/wiki/Cord_blood. [accessed on 15. 3. 2011].

11. http://stemcells.nih.gov/info/basics/basics5. [accessed on 20. 4. 2011].

12. http://learn.genetics.utah.edu/content/tech/ stemcells/sctoday/ [accessed on 28. 4. 2011].

13. http://www.LLS.org. [accessed on 1. 5. 2011].

14. http://cordblood.com/. [accessed on 25. 3. 2011]. 